Considering the vast number of optically and electronically active organic molecules, the organic version of the nanowires could reasonably be expected to have similar potential - and indeed, they are already being considered for devices such as transistors, LEDs and photovoltaic cells. Their onedimensional nanostructures offer the additional advantage of being mechanically flexible, making them particularly appealing for flexible optoelectronic applications: a hexathiapentacene nanowire transistor, for instance, suffers no significant loss in performance when placed under mechanical stress ${ }^{5}$.

Just as with their inorganic counterparts, several important optical properties have already been demonstrated in organic nanowires, including lasing ${ }^{7}$, waveguiding ${ }^{8}$, nonlinear optical mixing 9 and polarized emis$\operatorname{sion}^{10}$. With Zhao et al. ${ }^{1}$ adding colour tunability to the list, the nanowires seem to have a bright, white future ahead.

Melissa Fardy and Peidong Yang are in the

Department of Chemistry, University of

California, Berkeley, California 94720, USA.

e-mail: p_yang@berkeley.edu
1. Zhao, Y. S. et al. Adv. Mater. 20, 79-83 (2008).

2. Sun, M., Pullerits, T., Kjellberg, P., Beenken, W. J. D. \& Han, K J. Phys. Chem. A 110, 6324-6328 (2006).

3. Kuykendall, T., Ulrich, P., Aloni, S. \& Yang, P. Nature Mater. 6, 951-956 (2007)

4. Tang, C. W., VanSlyke, S. A. \& Chen, C. H. J. Appl. Phys. 65 , 3610-3616 (1989).

5. Briseno, A. L. et al. Nano Lett. 7, 668-675 (2007)

6. Xia, Y. et al. Adv. Mater. 15, 353-389 (2003)

7. O'Carroll, D., Lieberwirth, I. \& Redmond, G. Nature Nanotechnol. 2, 180-184 (2007).

8. Takazawa, K., Kitahama, Y., Kimura, Y. \& Kido, G. Nano Lett. 5, 1293-1296 (2005).

9. Brewer, J., Schiek, M., Lützen, A., Al-Shamery, K. \& Rubahn, H.-G. Nano Lett. 6, 2656-2659 (2006)

10. Datar, A. et al. J. Phys. Chem. B 110, 12327-12332 (2006).

\title{
pausranscrence
}

\section{Under Jupiter's pulsing skin}

\author{
Kunio M. Sayanagi
}

\section{Fast jet streams blow along the hallmark coloured bands that engirdle Jupiter's surface. By observing how storms erupt in these jet streams and disturb them, we can penetrate deeper into what lies beneath.}

Just as physicians use outward signs to diagnose the conditions inside their patients, so planetary scientists get beneath the skin of their equally dynamic subjects by looking at their surface appearances. Penetrating deep into Jupiter, the Solar System's largest planet, is especially difficult, as a thick cloud-deck keeps the lower levels of the atmosphere hidden from telescopic observations. Thus, SánchezLavega et al. (page 437 of this issue $)^{1}$ study the behaviour of giant storms at Jupiter's visible surface to reveal the vertical wind and temperature structure in the atmosphere beneath.

The vivid stripes of Jupiter make the planet one of the most visually appealing objects in our Solar System. Observations by NASA's two Voyager spacecraft, which flew past in 1979 , revealed that these bands of cloud are associated with atmospheric jet streams ${ }^{2}$. These jet streams are remarkably stable compared with their counterparts on Earth, which continually change course and vary in speed. Jupiter's jet streams, by contrast, flow essentially along lines of constant latitude. Measurements made by the Cassini probe $^{3}$, passing in 2000 on its way to Saturn, show no change in the jets' large-scale structure since the Voyager observations, and only minor differences in their locations and peak speeds.

Below this thick deck of cloud, however, observational records become scarce. The only direct measurements so far were made by the Galileo mission's entry probe ${ }^{4,5}$ in 1995 . This probe was designed to reach 140 kilometres below the clouds, but in fact entered a small, anomalously cloud-free region. Applying the

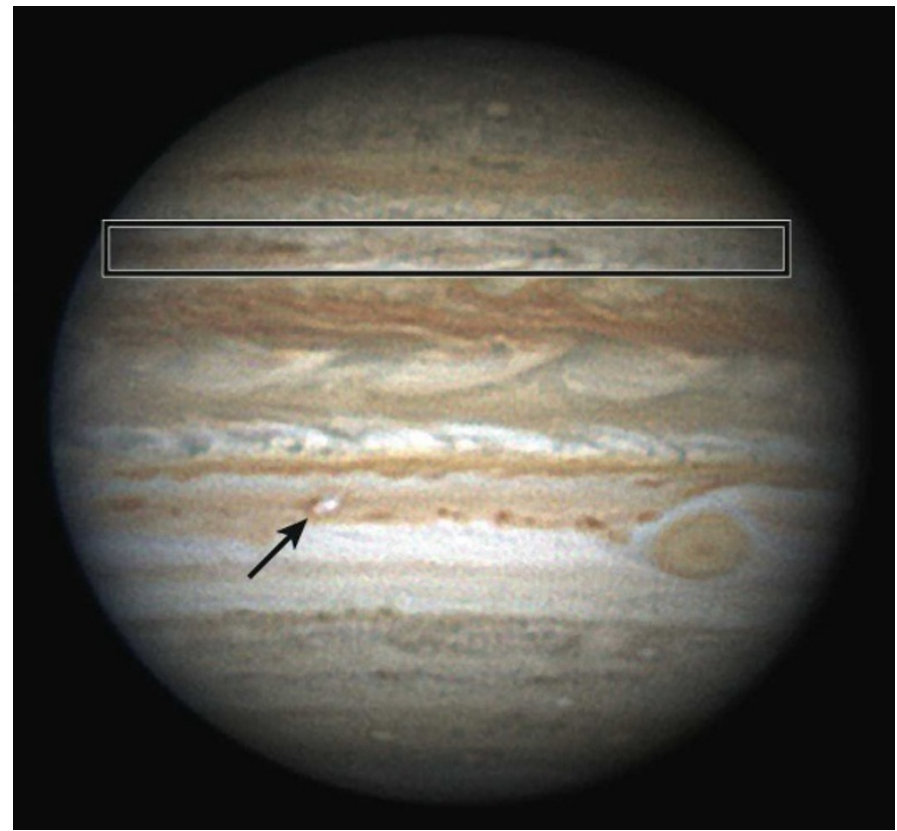

Figure 1 | Storm on camera. An image of Jupiter, obtained on 17 May 2007 from the island of Cebu in the Philippines by an amateur astronomer, shows the planet at the height of its global upheaval of that year. The box indicates the region of the $23^{\circ} \mathrm{N}$ jet, with the dark grey patches inside it representing the remnants of the disturbance triggered by the storms studied by SánchezLavega and colleagues ${ }^{1}$. The bright spot indicated by the arrow is the onset of yet another storm. The frequency and flexibility of amateur observations, coupled with the ability to achieve spatial resolutions high enough to resolve many major atmospheric features, has made them an integral part of Jupiter observation campaigns. (Image courtesy of C. $\mathrm{Go}^{1,13}$.) information gleaned from this single in situ measurement to the rest of the planet is therefore not straightforward.

As part of a campaign of observations to coincide with the passing of NASA's Pluto mission New Horizons, which swung by Jupiter in early 2007, Sánchez-Lavega et al. ${ }^{1}$ used the Hubble Space Telescope to monitor the Jupiter system. By chance, in late March 2007 they captured the onset of two large convective storms in a jovian jet stream at a latitude of $23^{\circ} \mathrm{N}$. This jet stream is particularly notable because it is Jupiter's fastest, with a speed that has varied between 140 and 180 metres per second in recent years $^{6-8}$.

The authors monitored the development of the storms using various ground-based telescopes as well as Hubble. The motion of the clouds during the outbursts gives useful hints about the atmospheric structures that lie beneath. In analysing their images, the authors found that the convective plumes substantially overshot the tropopause, a horizontal boundary in the atmosphere located above most clouds that generally acts as a stable dynamical lid on weather phenomena. The storm plumes must be extremely energetic, as they seem to extend more than 100 kilometres vertically from the storm's base below the thick clouddeck. Towers of cumulus cloud on Earth extend up to only about 10 kilometres or so in the vertical.

To explore this observation further, Sánchez-Lavega et al. ${ }^{1}$ performed numerical simulations with various background thermal stratifications. They show that, for a storm to become as energetic as those observed, the temperatures below the tropopause must be 2-5 kelvin colder than those measured by the Cassini probe on its $2000 \mathrm{fly}$ by $^{9}$. It is as yet unclear whether this difference represents a local spatial variance or a change in the vertical temperature structure throughout the latitude band over time. But constraining the thermodynamic 
conditions that allow episodic stormy outbursts is in itself a significant advance.

The authors' analysis also shows that the jet at $23^{\circ} \mathrm{N}$ remained robust throughout the storm disturbances. Using another numerical model, Sánchez-Lavega et al. tested various shapes of vertical shear below the clouds to reproduce the observed jet behaviour, and concluded that the jet stream's speed must increase with depth. This conclusion reaffirms an earlier result ${ }^{10}$ reached following studies of a very similar disturbance in 1990.

The disturbance in the $23^{\circ} \mathrm{N}$ jet marked the climax of a global upheaval on Jupiter, with changes in the colours of the visible clouds in several different latitude bands being seen throughout the 2007 observation season. This turmoil followed a very similar pattern to events in 1975 and 1990 (ref. 11). It is tempting to draw an analogy to a beating pulse - albeit an irregular one, and of unknown origin. It will be interesting to see whether a similar phenomenon returns sometime after 2020, following another break of a similar length. Saturn, too, has recurring giant convective outbursts ${ }^{12}$; both Jupiter and Saturn radiate more heat to space than they receive from the Sun, with cumulus convection transporting a great deal of heat vertically in their tropospheres. The pulsing nature of the giant storms on Jupiter and Saturn might even help us to understand how the heat flows from the deep interior to the surface on these planets.

A remarkable aspect of Sánchez-Lavega and colleagues' report ${ }^{1}$ is the increasing part now being played by amateur astronomers in planetary observation campaigns. The almost continuous observational coverage by amateurs around the globe allowed the growth and motion of individual large-scale storm clouds to be followed for up to 45 days, while the Hubble telescope performed occasional tracking of fine-scale features for about 10 hours at a time. In recent years, inexpensive digital cameras and access to sophisticated image-processing techniques that correct for the blurring caused by Earth's atmosphere have enabled advanced amateurs with modest-sized telescopes to image planets with resolutions high enough to resolve many atmospheric phenomena (Fig. 1). This coverage from around the world nicely complements the more powerful, but less flexible capabilities of the large ground- and space-based telescopes. Undoubtedly, as the planetary physicians continue to probe deeper under the skin of their subjects, the dedicated amateur medic will be on hand to supply some of the diagnostic tools.

Kunio M. Sayanagi is in the Comparative

Planetology Laboratory, University of Louisville,

Louisville, Kentucky 40292, USA.

e-mail: kunio.sayanagi@louisville.edu

1. Sánchez-Lavega, A. et al. Nature $\mathbf{4 5 1 , 4 3 7 - 4 4 0}$ (2008).

2. Limaye, S. S. Icarus 65, 335-352 (1986).

3. Porco, C. C. et al. Science 299, 1541-1547

(2003).
4. Atkinson, D. H., Pollack, J. B. \& Seiff, A. Science 272, 842-843 (1996).

5. Seiff, A. et al. Science 272, 844-845 (1996).

6. Rogers, J. H., Metting, H.-J. \& Peach, D. Icarus 184, 452-459 (2006)

7. Flasar, F. M. et al. Nature 427, 132-135 (2004)

8. Simon, A. A. Icarus 141, 29-39 (1999).
9. García-Melendo, E. \& Sánchez-Lavega, A. Icarus 152, 316-330 (2001)

10. García-Melendo, E. et al. Icarus 176, 272-282 (2005)

11. Rogers, J. H. The Giant Planet Jupiter (Cambridge Univ. Press, 1995).

12. Sánchez-Lavega, A. Chaos 4, 341-353 (1994).

13. www.christone.net/astro/jupiter

\section{MATERIALS SCIENCE \\ Designer pores made easy}

Michael J. Zaworotko

\section{Imagine being able to tweak the properties of a compound simply by replacing a molecular 'cartridge' with a different one. Just such a capability has been developed in a new class of porous crystalline materials.}

Porous materials are creating quite a stir among materials scientists because of their many possible uses, which range from gas storage to drug delivery. But before certain applications are possible, a simple way must be found of tweaking the properties of these molecular sponges. Reporting in the Journal of the American Chemical Society, Kawano et al. ${ }^{1}$ describe just such a method. They have prepared a porous material consisting of two molecular components, one of which can be easily replaced, rather like changing the cartridge in a pen. This flexibility allows the absorption properties of the solid to be fine-tuned.

Metal-organic materials are porous compounds consisting of metals or metal clusters bound to organic molecules known as ligands. Research into these compounds has undergone two bursts of growth over the past couple of decades. The first occurred after the publication of a seminal paper ${ }^{2}$ in 1990 , which outlined

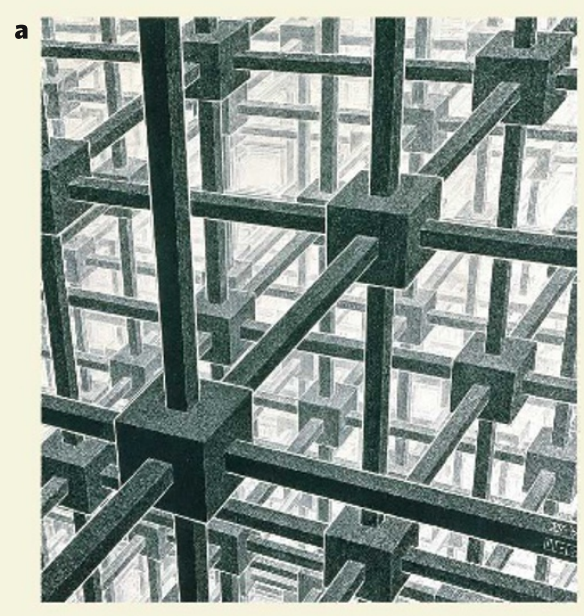

the design opportunities these compounds present for controlling the arrangements of atoms in solids. The second burst occurred in the late 1990s, when it became clear that such compounds combine unprecedented levels of porosity with properties such as magnetism, catalysis, polarity and luminescence ${ }^{3-5}$. This makes them potentially useful for many applications, including for gas storage and separation, as chemical and biological sensors, and even for harvesting energy from light.

Metal-organic materials now deservedly lie at the forefront of advanced materials, offering a synergistic suite of features that gives them several advantages over other porous compounds. Their first useful property is ease of design. Most crystal structures are unpredictable, but those of metal-organic materials are controllable. They also have structural blueprints similar to those found in nature. For example, they can exist as 'zero-dimensional' nanostructures based on atomic polyhedra, rather like those

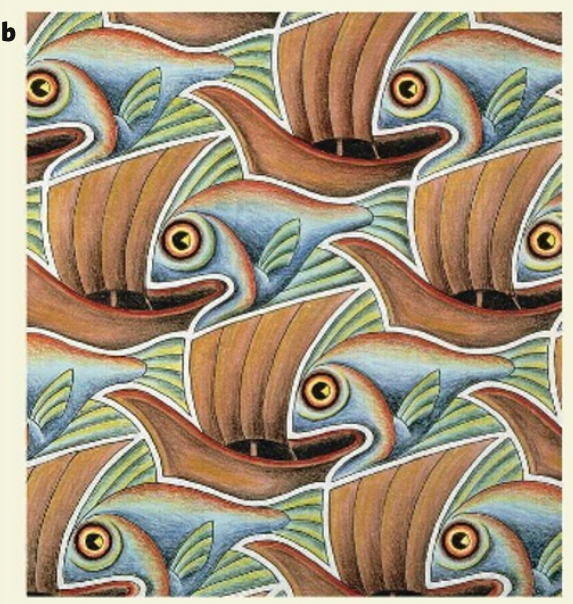

Figure 1 | Blueprints for form and function in porous solids. Metal-organic materials are porous crystalline solids, aspects of which are depicted in these paintings by M. C. Escher. a, Cubic Space Division (1952) shows a 'cubic net' framework. Some metal-organic materials adopt this structure, in which each cube is replaced with an appropriate molecular building-block. b, In Symmetry E72 (Fish and Boats) (1949), the spaces between the boats match the shape of the fish exactly. Similarly, metalorganic materials can be designed so that their pores fit exactly around specific target molecules - a prerequisite for molecular recognition. 